\title{
Non-Binary N-MSK non-orthogonal Modulation for wireless communication
}

\author{
Jie Shen ${ }^{1 \mathrm{a}^{*}}$, Jianguo $\mathrm{Yu}^{2 \mathrm{~b}}$ and Lun Zhao, Lei Chen ${ }^{2}$ \\ ${ }^{1}$ China potevio., Co Ltd, Beijing, P.R.China 100080;'Dept of Electronics, Beijing Univ of PostS \& \\ Telecomnd Beijing, P.R.China 100876 \\ ashenjie75@sohu.com, byujg@bupt.edu.cn
}

Keywords: Non-binary; No-orthogonal; N-MSK; TCM; Ring Integers; MCE; Genetic Algorithm

\begin{abstract}
This paper presents a novel multi-level convolutional encoder(MCE) modulation method, which is non-binary TCM based on Rings of Integers. We also give how to searching such good codes, which can be decoded by genetic algorithm. Through simulation different environments, the paper shows such codes have advantage over traditional TCM.
\end{abstract}

\section{Introduction}

Convolutional codes based on rings of integers modulo-M were first presented by Massey and Mittelholzer [1, 2]. They were followed by Baldini and Farrell [3, 4], who presented TCM codes based on rings of integers. Baldini and Farrell have developed a number of modulo-M ring-TCM codes for N-PSK constellations and have concluded that, due to the similarities between N-MSK signal sets and the algebraic structure of rings of integers modulo- $\mathrm{M}$, modulo-M ring-TCM codes are the natural linear codes for N-MSK modulation.

In this paper, we propose a new overlapped non-orthogonal modulation theory, based on the theory apply the N-MSK for wireless communication system. We also present the NMSK coding based on Rings of Integers, and how to search such code. Then a novel genetic decoding algorithm are discussed in detail, its performance are evaluated through simulation.

Through studying its performance under different channel circumstance, we learned the N-MSK has better SIR characters, and higher spectrum efficiency than traditional TCM modulation.

At last, we also extend the application to non-orthogonal situation, which can be used for improving the performance of wireless system.

\section{N-MSK modulation model}

NMSK signal are consisting of N MSK signals with different amplitudes, which can be expressed as[12]:

$$
S_{N M S K}\left(t, \alpha_{1}, \alpha_{2}, \ldots, \alpha_{N}\right)=A \sum_{i=1}^{N} S\left(t, \alpha_{i}\right) \quad \text { (1a) } A=\sqrt{2 E / T} / \sqrt{\sum_{i=1}^{N} 2^{2(i-1)}}
$$

Where $E$ presents average engorge; and $T$ presents code interval; $S\left(t, \alpha_{i}\right)$ presents the $i$ th componentsof NMSK, which can be expressed as

$$
\begin{aligned}
S\left(t, \alpha_{i}\right)=2^{i-1} \cos \left(2 \pi f_{c} t+\frac{\pi \alpha_{i, n}(t-(n-1) T)}{2 T}+\phi_{i, n}\right) & \text { (2a) } \phi_{i, n}=\frac{\pi}{2} \sum_{j=0}^{n-1} \alpha_{i, j}+\phi(0) \\
(n-1) T & \leq t<n T
\end{aligned}
$$


Here, $\alpha_{i, n}$ is the $i$ th MSK signal components imputed during the $n$th timeslot and $\alpha_{i, n} \in\{ \pm 1\}$; $\phi(0)$ is the initial phase, in general, let $\phi(0)=0$. Meanwhile, MSK equals to concatenation of two components: Convolute process entity (CPE) and memory-less waveform mapping entity.

MSK input and output be presented as:

$$
S(t)=\left(U_{1, n} \cos 2 \pi f_{1} t+U_{2, n} \cos 2 \pi f_{2} t\right) g(t-n T)
$$

where $f_{2}-f_{1}=1 / 2 T$ and $f_{1}=f_{c}-1 / 4 T$. So, NMSK be represented as:

$$
S_{\text {NMSK }}(t)=\left(W_{1, n} \cos 2 \pi f_{1} t+W_{2, n} \cos 2 \pi f_{2} t\right) g(t-n T) \quad(4 \mathrm{a}) \quad\left(W_{1 n}, W_{\Omega}\right)=A\left(\sum_{i=1}^{N} 2^{i-1} U_{1 n}^{x}, \sum_{i=1}^{N} 2^{i-1} U_{2 n}^{i}\right)
$$

\section{TCM Based on Rings of Integers}

Assuming that $\mathrm{m}$ information bits are transmitted per baud, the general structure of a ring-TCM encoder suitable for N-MSK modulation, with $M=2^{m+1}$, is shown in Figure 1. This ring-TCM encoder works as follows: first, $m+1$ information bits, $b_{i}$, are mapped into a modulo-M symbol, $a_{j}$, according to a mapping function $\mathrm{f}$ (for instance, $f$ can be a Gray mapping function). Next, $m$ modulo-M $a_{j}$ symbols are introduced into a linear multi-level convolutional encoder (MCE), which generates $m+1$ modulo-M coded symbols, $x_{k}$. Finally, each one of these coded symbols $x_{k}$ is associated with a signal of the N-MSK signal set and is sent to the channel. Ahs a total of $m+1$ modulo-M coded symbols $x_{k}$ are transmitted per single trellis branch, ring-TCM codes can be considered as $2(m+1)$-dimensional TCM codes.

Propagation delays caused by a fading channel can result in phase shifting of the transmitted signal. At the demodulator these phase shifts result in a rotation of the received signals compared with the transmitted symbols. If, after these phase shifts, the received word is another valid code word, that code is known as phase invariant.

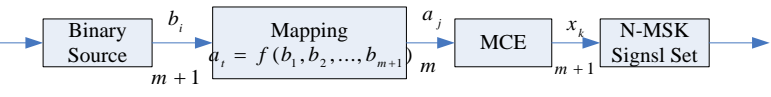

Figure 1. General structure of a ring-TCM encoder suitable for N-MSK modulation.

A multi-level convolutional code is $360 / M$ phase invariant if and only if the all-one code word can be found in the code [4]. A code with the all-one code word is said to have a transparent encoder [4]. For example, an MCE defined over $\mathbf{Z}_{4}$ is $360 / 4=90 \circ$ phase invariant if the all-one code word is present in the code.

In general, transparent modulo-M ring-TCM codes can be readily designed, with neither additional difficulty nor significant decrease in performance with respect to nontransparent ring-TCM codes. Furthermore, in addition to the transparency property, ring-TCM codes present, in general, better coding gains than their nontransparent Ungerboeck counterparts. However, the major drawback of these codes is that they generally require a more computationally intensive decoding process. Therefore, although it has already been established that ring-TCM schemes can constitute a powerful alternative to conventional TCM schemes, their actual performance/complexity tradeoff should be investigated.

There is also the necessity to extend the use of ring-TCM codes to other modulation schemes than $\mathrm{N}$-MSK constellations. In particular, the structure of a ring-TCM encoder based on rings of integers modulo-4, which is suitable for any rectangular M-QAM constellation, has been proposed [4]. This novel coded modulation scheme can result in ring-TCM codes with excellent coding gains which are, in addition, transparent to phase rotations of $360 / 4=90$. 


\section{Genetic Algorithm}

The choice of feed-forward and feedback coefficients of the MCE can significantly change the parameters of the code, such as the free distance, $d_{\text {free }}$, and so it is important to have methods for finding good TCM codes. The most obvious method would be to apply an exhaustive search algorithm where all values of the coefficients are tried, but this would become too time-consuming for larger codes defined over larger rings.

There are two criteria for good TCM codes over a Gaussian channel: maximizing the free distance and minimizing the number of paths in the code trellis with Euclidean distance equal to the free distance, denoted as $N_{\text {free }}$, subject to the feed-forward and feedback coefficients [9]:

$$
\max d_{\text {free }}\left\{g_{s}^{(i)}, \ldots, g_{0}^{(i)}, f_{s}, \ldots, f_{1}\right\} \quad \text { (5) } \quad \min N_{\text {free }}\left\{g_{s}^{(i)}, \ldots, g_{0}{ }^{i(}, f_{s}, \ldots, f_{1}\right\}
$$

In the following section, a much faster search algorithm for finding good ring-TCM codes for N-MSK and M-QAM is presented.

For the solution of optimization problems, a genetic algorithm [10] has been investigated for several applications in signal processing, such as speech compression, and has been shown to be effective at exploring a large and complex search space in an adaptive way. Next, a formal definition of genetic algorithms is given, and the operators used in this work are described.

A genetic algorithm can be defined as the nine-tuple:

$\mathrm{GA}=\left\{p^{0}, S, \lambda, L, f, s, c, m, T\right\}$, where: $p^{t}=\left\{a_{1}^{t}, \ldots, a_{\lambda}^{t}\right\}=$ population in the $t$ th generation; $S=$ search space in which the chromosome is encoded; $a_{\lambda}^{0} \in S=$ elements of the search space: $\lambda=$ population size; $L=$ length of chromosome; $f: S \rightarrow R=$ fitness function; $s: S^{\lambda} \rightarrow S=$ selection operator; $c: S \times S \rightarrow S \times S=$ crossover operator ; $m: S \rightarrow S=$ mutation operator; $T: S^{\lambda} \rightarrow\{0,1\}=$ termination criterion.

In searching for TCM codes, the genetic algorithm basically selects and classifies the generator code word with a high degree of adaptation as parents generate a new generation by the combination of their components, and by the elimination of the weakest generators from the population [11]. In genetic algorithms there are many operators but the selection, crossover and mutation operators are only used for code searching.

Selection Operator. Assuming that the initial population $p^{0}=\left(a_{1}^{0}, \ldots, a_{\lambda}^{0}\right)$ can be obtained in a heuristic or random way from the search space, $\mathrm{S}$, in which the chromosome is encoded, the next generation is obtained from members of the previous generation using a stochastic process, which guarantees that the number of times that one structure is selected is dependent on its performance compared with the rest of the population.

The parent-selection operation $s, s: S^{\lambda} \rightarrow S$ produces an intermediate population $p^{t}=\left(a_{1}^{t}, \ldots, a_{\lambda}^{t}\right)$ from the population $p^{t}=\left(a_{1}^{t}, \ldots, a_{\lambda}^{t}\right)$ in the $t$ th generation, $s\left(p^{t}\right)=p^{t}$. Any at $a_{i}^{t}=a_{q}^{t}$ in 
the $p^{t}$ is selected by a given random number $\alpha_{i}$ satisfying the

following condition:

$$
0 \leq \alpha_{i} \leq \sum_{j=1}^{\lambda} f\left(a_{j}^{t}\right) \quad \text { (7) } \quad q=\mathrm{m} \mathrm{a}\left\{\mathrm{k} / \forall k \in\{\ldots 1 \lambda\} s t, \alpha_{i} \leq \sum_{j=1}^{\lambda} . f a_{j}^{t}\right\}
$$

The index $q$ is obtained from (8), where $\sum_{j=1}^{\lambda} f\left(a_{j}^{t}\right)$ is the summation of all the fitness from population $p^{t}$ for all members of the population picking up the first index $k$ that reaches $\alpha_{i} \leq \sum_{j=1}^{\lambda} f\left(a_{j}^{t}\right) \cdot$

Crossover Operator. For any selected chromosomes in a population $p^{t}$, an associated real value $0 \leq \rho \leq 1$ is generated randomly. If $\rho$ is greater than the defined crossover threshold $\rho_{c}$ where $0 \leq \rho_{c} \leq 1$, the crossover operator $c: S \times S \rightarrow S \times S$ is applied to this pair of chromosomes. The strategy used in this work is the one-point crossover, cop, which produces an intermediate population pt from the population pt and is defined below:

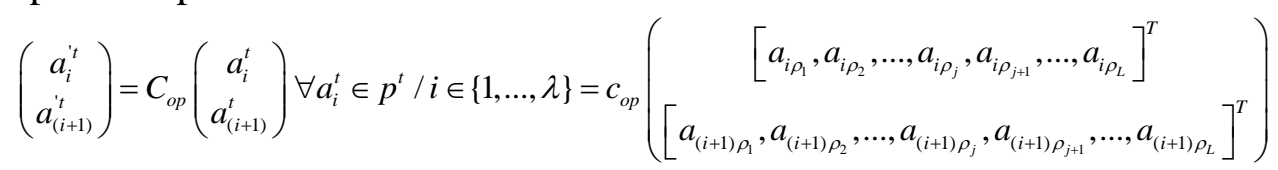

The pair of chromosomes is separated into two subchromosomes at $\rho_{j}$, hence a new pair is composed by swapping the second subchromosome, where each one is crossed from $\rho_{j+1}$ to $\rho_{L}$.

Mutation Operator. For any chromosome in a population $p^{t}$, an associated real value $0 \leq \rho \leq 1$ is generated randomly. If $\rho$ is less than the defined mutation threshold $\rho_{m}$, where $0 \leq \rho_{m} \leq 1$, the mutation operator is applied to the chromosome. The mutation operator simply alters one bit in a chromosome from 0 to 1 (or 1 to 0 ). The mutation operator $m: S \rightarrow S$ produces an intermediate population $p^{t}$ from the population $p^{t}$, as below:

$$
a_{i}^{t^{t}}=m\left(a_{i}^{\prime t}\right) \quad \forall_{i} \in\{1, \ldots, \lambda\} \quad a_{i, \rho k}^{t^{t}}= \begin{cases}a_{i, \rho k} & \text { for } k \in\{1,2, . p-, \quad p+1, \quad L\} \\ \bar{a}_{i, \rho k} & \text { for } k=p\end{cases}
$$

The reproduction and crossover operators give to the genetic algorithms the bulk of their processing power. The mutation operator is needed because, even though reproduction and crossover are the main operators, occasionally they may become overzealous and lose some potentially useful genetic material. 
Usually there are four parameters to control the evolution of the genetic algorithm. They are: the population size, $\lambda$; the crossover threshold, $\rho_{c}$; the mutation threshold, $\rho_{m}$; and the number of generations. If three of these are kept fixed, an optimum value for the free parameter can be found in order to produce the optimum code rate, $d_{\text {free }}$ and $N_{\text {free }}$.

The disadvantage of the exhaustive search algorithm is the time taken to generate codes, especially when a high number of states and a high-order constellation are used. It is important to note that the exhaustive search for the rectangular M-QAM constellation is more computationally intensive than that for N-MSK constellations, because the rectangular M-QAM constellation requires that, to determine $d_{\text {free }}$ and $N_{\text {free }}$, all paths in the trellis be examined and compared to each other. The fitness function used in this work is an a priori function based on the computation of the distances.

Once the code is obtained, a further check is required to investigate the performance in terms of symbol error rate as a function of signal-to-noise ratio. The application of the genetic algorithm to 16-QAM is shown with new codes for this particular modulation scheme to demonstrate its feasibility, when the number of states is increased to produce better constraint lengths. The application of the genetic algorithm to the code-to-signal mapping for 16-QAM has produced a range of $180^{\circ}$ and $90^{\circ}$ rotational invariant ring-TCM codes (RI ring-TCM) [11].

For convenience, we denote $d_{\text {free }}^{2}$ is the minimum squared Euclidean distance between coded sequences of the ring-TCM code suitable for 16-QAM; $g_{\infty}$ is the asymptotic coding gain of the ring-TCM code suitable for16-QAMover uncoded8-AMPMmodulation; *means that the exhaustive search was stopped; and means a new generated code using the genetic algorithm.

\section{Performance of Ring-TCM Codes on Urban Fading Channels}

Three simulation results are presented, showing the performances of three ring-TCM codes on an indoor, a pedestrian and a vehicular channel. The ring-TCM codes tested were transparent, and decoded using the soft-decision Viterbi algorithm. The codes used were the 4-state 21/2, 8-state 213/30 and 16-state 212/31 ring-TCM codes defined over $\mathbf{Z}_{4}$. The user velocities were $0 \mathrm{mph}$ for indoor, $4 \mathrm{mph}$ for pedestrian and $70 \mathrm{mph}$ for vehicular. The channel scenarios are easily modified by altering the delay, power profile and Doppler spectra to create virtually any single input-single-output (SISO) environment based on measured data. This gives a much more flexible channel model, which corresponds to actual measured data and produces a time-varying frequency-selective channel that is much more realistic and is essential for testing certain distortion mitigation techniques.

Figure 2 shows the performance of the ring-TCM codes on the indoor channel. This is a slow-fading channel, but the least harsh of the three urban channel models and good results are achieved, with the 16-state 212/31 ring-TCM code achieving a coding gain of $1.2 \mathrm{~dB}$ over the $21 / 2$ and 213/30 ring-TCM codes. Figure 3 shows the performance of the ring-TCM codes on the pedestrian channel. This channel is more harsh but the relative performance of the three ring-TCM 
codes has not changed. The 212/31 ring-TCM code achieves a coding gain of approximately $14 \mathrm{~dB}$ over uncoded performance. Figure 4 shows the performance of the ring-TCM codes on the vehicular channel. This channel is a time-varying fading channel and is very harsh. The performance of all three ring-TCM codes is poor and there is no significant coding gain over an uncoded system.

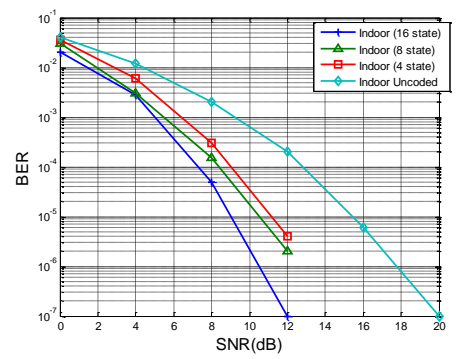

Figure 2. Ring-TCM codes on the indoor channel.

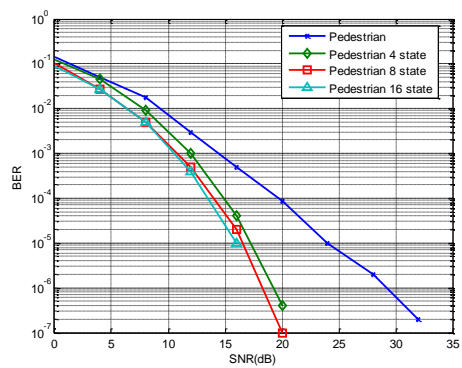

Figure 3. Ring-TCM codes on the pedestrian channel.

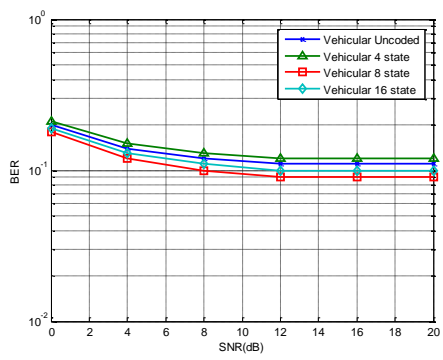

Figure 4. spectrum evaluation of extend LS code

\section{Acknowledgements}

The authors would like to acknowledge the comments of colleagues, and the support of Chinese NSF 61372035

\section{References}

1. Massey, J.L. and Mittelholzer, T. (1984) Codes over rings - a practical necessity. Presented at AAECC7 International Conference, Universite' P. Sabatier, Toulouse, France.

2. Massey, J. and Mittelholzer, T. (1989) Convolutional codes over rings. Proceedings of the 4th Joint Swedish Soviet Workshop Information Theory, pp. 14-8.

3. Baldini, R.F. and Farrell, P.G. (1990) Coded modulation wit convolutional codes over rings. Presented at Second IEE Bangor Symposium on Communications, Bangor, Wales.

4. Baldini, R.F. and Farrell, P. (1994) Coded modulation based on rings of integers modulo-q part 2: convolutional codes. IEE Proceedings: Communications, 141, 137-42.

5. Carrasco, R., Lopez, F. and Farrell, P. (1996) Ring-TCM for N-MSK modulation: AWGN channels and DSP implementation. Communications, IEE Proceedings, 143, 273-80.

6. Carrasco, R. and Farrell, P. (1996) Ring-TCM for fixed and fading channels: land-mobile satellite fading channels with QAM. Communications, IEE Proceedings, 143, 281-8.

7. Lopez, F.J., Carrasco, R.A. and Farrell, P.G. (1992) Ring-TCM Codes over QAM. IEE Electronics Letter, 28, 2358-9.

8. Tarokh, V., Seshadri, N. and Calderbank, A. (1998) Space-time codes for high data rate wireless communication: performance criterion and code construction. Information Theory, IEEE Transactions on, 44, 744-65.

9. Benedetto, S., Mondin, M. and Montorsi, G. (1994) Performance evaluation of trellis-coded modulation schemes. Proceedings of the IEEE, 82, 833-55.

10. Goldberg, D. (1989) Genetic Algorithms in Search, Optimization and Machine Learning, Addison-Wesley Longman Publishing Co., Inc., Boston, MA, USA.

11. Soto, I. and Carrasco, A. (1997) Searching for TCM codes using genetic algorithms. 
Communications, IEE Proceedings, 144, 6-10.

12. Javornik T, Kandus G, Burr A. The performance of N-MSK signals in non-linear channels [J]. IEICE Transactions on Communications, 2002, E85-B: 1265-1275. 